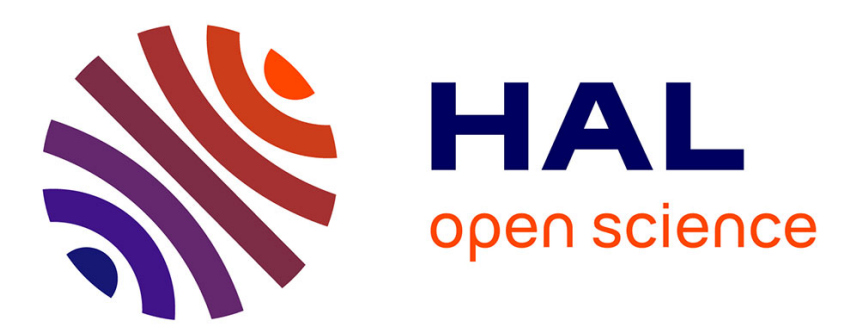

\title{
Constraint Programming Approach to the Problem of Generating Milton Babbitt's All-Partition Arrays
}

\author{
Tsubasa Tanaka, Brian Bemman, David Meredith
}

\section{To cite this version:}

Tsubasa Tanaka, Brian Bemman, David Meredith. Constraint Programming Approach to the Problem of Generating Milton Babbitt's All-Partition Arrays. The 22nd International Conference on Principles and Practice of Constraint Programming, Sep 2016, Toulouse, France. 10.1007/978-3-319-449531_50. hal-01467879

\author{
HAL Id: hal-01467879 \\ https://hal.science/hal-01467879
}

Submitted on 14 Feb 2017

HAL is a multi-disciplinary open access archive for the deposit and dissemination of scientific research documents, whether they are published or not. The documents may come from teaching and research institutions in France or abroad, or from public or private research centers.
L'archive ouverte pluridisciplinaire HAL, est destinée au dépôt et à la diffusion de documents scientifiques de niveau recherche, publiés ou non, émanant des établissements d'enseignement et de recherche français ou étrangers, des laboratoires publics ou privés. 


\title{
Constraint programming approach to the problem of generating Milton Babbitt's all-partition arrays
}

\author{
Tsubasa Tanaka ${ }^{1}$, Brian Bemman ${ }^{2}$ and David Meredith ${ }^{2}$ \\ 1 STMS Lab : IRCAM, CNRS, UPMC, Paris, France \\ tsubasa.tanaka@ircam.fr \\ 2 Aalborg University, Aalborg, Denmark \\ $\{\mathrm{bb}$, dave $\}$ @create. aau. $\mathrm{dk}$
}

\begin{abstract}
Milton Babbitt (1916-2011) was a composer of twelve-tone serial music noted for creating the all-partition array. One part of the problem in generating an all-partition array requires finding a covering of a pitch-class matrix by a collection of sets, each forming a region containing 12 distinct elements and corresponding to a distinct integer partition of 12 . Constraint programming (CP) is a tool for solving such combinatorial and constraint satisfaction problems. In this paper, we use CP for the first time to formalize this problem in generating an allpartition array. Solving the whole of this problem is difficult and few known solutions exist. Therefore, we propose solving two sub-problems and joining these to form a complete solution. We conclude by presenting a solution found using this method. Our solution is the first we are aware of to be discovered automatically using a computer and differs from those found by composers.
\end{abstract}

Keywords: Babbitt · all-partition array · computational musicology · constraint programming

\section{Introduction}

Milton Babbitt (1916-2011) was a composer of twelve-tone serial music noted for developing highly constrained and often complex musical structures. Many of his pieces are organized according to one such structure known as the allpartition array [1]. An all-partition array is a covering of a matrix of pitch-class integers by a collection of sets, each of which forms a region in this matrix containing 12 distinct pitch classes from consecutive elements in its rows and that corresponds to a distinct integer partition of 12 (to be clarified in the next section). This unique structure imposes a strict organization on the pitch classes in his works, and it serves as both a method of musical composition and musical form. Moreover, the all-partition array allowed Babbitt one of many ways to achieve maximal diversity in his music. ${ }^{3}$

${ }^{3}$ Maximal diversity is the presentation of as many musical parameters in as many different ways as possible [2]. 
In this paper, we formulate one part of the problem in generating an allpartition array, beginning from a given matrix of pitch-class integers, using constraint programming $(\mathrm{CP})$ and with a particular focus on its mathematical aspects. Using our model and a method for dividing this matrix into smaller, sub-problems, we obtained a solution, which, we believe, is the first to be discovered automatically using a computer and differs from those found by composers. $\mathrm{CP}$ is a programming paradigm that has been successfully applied to the solving of various constraint satisfaction problems in music [3-7]. It seems natural then, that $\mathrm{CP}$ could be used in the problem we address here. Moreover, having such a model could, for example, be used as a basis for generating new musical works.

\subsection{The structure of an all-partition array}

In this section, we describe the structure of an all-partition array in a way that assumes the reader has a basic understanding of pitch class set theory. Constructing an all-partition array begins with the construction of an $I \times J$ matrix, $A$, whose elements are pitch-class integers, $0,1, \ldots, 11$, where each row contains $J / 12$ twelve-tone rows. The dimensions of this matrix constrain the most important requirement of the structure of an all-partition array, however, Babbitt generally limited himself to sizes of $4 \times 96,6 \times 96$, and $12 \times 72$ [2]. In this paper, we consider only matrices where $I=4$ and $J=96$, as matrices of this size figure prominently in Babbitt's music [2]. This results in a $4 \times 96$ matrix of pitch classes, containing 32 twelve-tone rows from the possible 48 related by any combination of transposition, inversion and retrograde (i.e., reversal). In other words, $A$ will contain an approximately uniform distribution of 32 occurrences of each of the integers from 0 to $11 .{ }^{4}$ On the musical surface, rows of this matrix become expressed as 'musical voices', typically distinguished from one another by instrumental register [2].

A complete all-partition array is a covering of matrix, $A$, by $K$ sets, each of which is itself a partition of the set $\{0,1, \ldots, 11\}$ whose parts (1) contain consecutive row elements from $A$ and (2) have cardinalities equal to the summands in one of the $K$ distinct integer partitions of 12 (e.g., $6+6$ or $5+4+2+1$ ) containing $I$ or fewer summands greater than zero. ${ }^{5}$ Figure 1 shows a $4 \times 12$ excerpt from a $4 \times 96$ pitch-class matrix, $A$, and two such sets forming regions in $A$ each containing every pitch class exactly once and corresponding to two distinct integer partitions, whose exact "shapes" are more precisely represented as the integer compositions, $\operatorname{IntComp}_{12}(4,4,4,0)$ and $\operatorname{IntComp}_{12}(0,6,3,3){ }^{6}$

\footnotetext{
${ }^{4}$ For a more detailed description of the constraints governing the organization of matrices in Babbitt's music, see [2,8].

${ }^{5}$ We denote an integer partition of an integer, $L$, by $\operatorname{IntPart}_{L}\left(s_{1}, s_{2}, \ldots, s_{I}\right)$ and define it to be an ordered set of non-negative integers, $\left\langle s_{1}, s_{2}, \ldots, s_{I}\right\rangle$, where $L=\sum_{i=1}^{I} s_{i}$ and $s_{1} \geq s_{2} \geq \cdots \geq s_{I}$.

${ }^{6}$ We define an integer composition of a positive integer, $L$, denoted by $\operatorname{IntComp}_{L}\left(s_{1}, s_{2}, \ldots, s_{I}\right)$, to also be an ordered set of $I$ non-negative integers, $\left\langle s_{1}, s_{2}, \ldots, s_{I}\right\rangle$, where $L=\sum_{i=1}^{I} s_{i}$.
} 


\begin{tabular}{|llll|llllllll}
\hline 11 & 10 & 3 & 7 & 6 & 2 & 4 & 5 & 8 & 1 & 9 & 0 \\
8 & 9 & 4 & 0 & 1 & 5 & 3 & 2 & 11 & 6 & 10 & 7 \\
2 & 5 & 1 & 6 & 9 & 10 & 0 & 8 & 7 & 11 & 4 & 3 \\
\cline { 1 - 10 } & 4 & 8 & 3 & 0 & 11 & 9 & 1 & 2 & 10 & 5 & 6
\end{tabular}

Fig. 1: A $4 \times 12$ excerpt from a $4 \times 96$ pitch-class matrix with two distinct integer partition regions represented precisely by the integer compositions, $\operatorname{IntComp}_{12}(4,4,4,0)$ (in dark gray) and $\operatorname{IntComp}_{12}(0,6,3,3)$ (in light gray), each containing every pitch class exactly once.

Note, in Figure 1, that each summand (from left to right) in $\operatorname{IntComp}_{12}(4,4,4,0)$, gives the number of elements in the corresponding row of the matrix (from top to bottom) in this region. Unlike common tiling problems using, for example, polyominoes, these regions need not have connected interiors, as demonstrated by the second region in Figure 1 between rows 3 and 4. On the musical surface, the distinct shape of each region helps contribute to a progression of 'musical voices' that vary in textural density, allowing for relatively thick textures in, e.g., $\operatorname{IntComp}_{12}(3,3,3,3)$ (with four participating parts) and comparatively sparse textures in, e.g., IntComp ${ }_{12}(11,0,1,0)$ (with two participating parts).

There exist a total of 34 distinct integer partitions of 12 into 4 or fewer non-zero summands [2]. An all-partition array with four rows will thus contain $K=34$ regions, each containing every pitch class exactly once and each with a distinct "shape" determined by an integer composition defining a distinct integer partition. However, the number of pitch classes required to satisfy this constraint, $34 \times 12=408$, exceeds the size of a $4 \times 96$ matrix containing 384 elements, by 24 . In order to satisfy this constraint, contiguous regions may share pitch classes, with the added constraint that only horizontal overlaps of at most one pitch class in each row are allowed for each of the 34 integer partition regions. Figure 2 shows a third region, $\operatorname{IntComp}_{12}(5,1,0,6)$ (in medium gray), in the matrix shown in Figure 1, where two of its elements result from overlapped pitch classes from previous regions. Note, in Figure 2, the two horizontal overlaps of pitch class, 7

\begin{tabular}{|llll|llll|lll|r|}
\hline 11 & 10 & 3 & 7 & 6 & 2 & 4 & 5 & 8 & 1 & 9 & 0 \\
8 & 9 & 4 & 0 & 1 & 5 & 3 & 2 & 11 & 6 & 10 & 7 \\
2 & 5 & 1 & 6 & 9 & 10 & 0 & 8 & 7 & 11 & 4 & 3 \\
\cline { 1 - 6 } & 4 & 8 & 3 & 0 & 11 & 9 & 1 & 2 & 10 & 5 & 6
\end{tabular}

Fig. 2: A $4 \times 12$ excerpt from a $4 \times 96$ pitch-class matrix with a third integer composition, $\operatorname{IntComp}_{12}(5,1,0,6)$ (in medium gray), sharing one pitch class from each of the two previous regions.

(in row 1 and belonging to the first region) and 8 (in row 4 and belonging to the second region), required to have each pitch class occur exactly once in the third 
integer partition region. This means that while contiguous regions may share pitch classes, such regions need not be necessarily adjacent in sequence.

Composers have primarily relied on constructing all-partition arrays by hand and at least some of their methods have been published $[1,9,10]$. Algorithms for automating this task have also been proposed $[8,11]$. However, generating an allpartition array is a large combinatorial problem and satisfying the constraints of its structure is difficult. To date, none of these algorithms have been able to solve this problem automatically. This observation motivates our decision here to look for alternative programming paradigms and methods for possibly better addressing this problem. In section 2, we present our CP constraints for implementing the problem of generating an all-partition array from a given matrix. As solving for the entire matrix directly is difficult, in section 3, we present a method of dividing this matrix into two smaller matrices, choosing integer partitions based on how frequently they appear in solutions to one of these smaller matrices, and re-joining them to form a complete solution. We conclude here with a solution discovered using this method.

\section{CP constraints for the problem of generating an all-partition array from a given matrix}

We begin the discussion of our $\mathrm{CP}$ constraints for generating an all-partition array, with a given matrix found in one of Babbitt's works based on the allpartition array. ${ }^{7}$ Let $\left(A_{i, j}\right)$ be this $(4,96)$-matrix whose elements are the pitchclass integers, $0,1, \ldots, 11$. We denote the number of rows and columns by $I$ and $J$, respectively. Let $x_{i, j, k}(1 \leq i \leq I, 1 \leq j \leq J)$ be a binary variable corresponding to each location $(i, j)$ in $A$ and a subset (i.e., a region) identified by the integer $k$, where $1 \leq k \leq K$ and $K=34$. There are then 34 sets of 384 such variables. Each of these variables will indicate whether or not a location $(i, j)$ belongs to a candidate set, which we denote, $C_{k}$, for the $k$ th position in the sequence of 34 regions. For $C_{k}$ to be a candidate set, it must form a region in $A$ (as described in section 1), by satisfying two conditions, consecutiveness and containment, which we will introduce below. Having satisfied these conditions, $C_{k}$ will be a candidate set in a possible solution to our problem, in which its elements correspond to 12 distinct pitch classes in $A$ and whose "shape" is defined by an integer composition. Additional constraints e.g., ensuring that each of these candidate sets is then a distinct integer partition and that their overlaps do not exceed one in each row, will then complete our formulation of this problem.

\subsection{Consecutiveness}

The condition of consecutiveness states that pitch classes belonging to the same region and row in $A$ must lie adjacent to one another with no gaps between. We

\footnotetext{
${ }^{7}$ Examples of this matrix can be found in Babbitt's My Ends are My Beginnings
} (1978) and Beaten Paths (1988), among others. 
ensure this is the case by placing constraints on the strings of 0's and 1's that are allowed in the rows formed by $\left\langle x_{i, 1, k}, x_{i, 2, k}, \ldots, x_{i, J, k}\right\rangle$ for each $(i, k)$. If, for example, the string $\langle\ldots, 0,1, \ldots\rangle$ appears in the $i$ th row for some $k$, then there can be no 1 occurring before 0 . This is expressed by the following:

$$
\begin{aligned}
\forall i \in[1, I], \forall j \in[3, J], \forall k \in[1, K] & \\
\left(x_{i, j-1, k}=0\right. & \left.\wedge x_{i, j, k}=1\right) \Longrightarrow \stackrel{\wedge_{j^{\prime}=1}^{j-2}\left(x_{i, j^{\prime}, k}=0\right) .}{ }
\end{aligned}
$$

On the other hand, if $\langle\ldots, 1,0, \ldots\rangle$ appears in this row, then there can be no 1 after 0 . This is expressed by the following:

$$
\begin{aligned}
& \forall i \in[1, I], \forall j \in[1, J-2], \forall k \in[1, K], \\
&\left(x_{i, j, k}=1 \wedge x_{i, j+1, k}=0\right) \Longrightarrow \bigwedge_{j^{\prime}=j+2}^{J}\left(x_{i, j^{\prime}, k}=0\right) .
\end{aligned}
$$

In other words, all 1's in $\left\langle x_{i, 1, k}, x_{i, 2, k}, \ldots, x_{i, J, k}\right\rangle$ for each $(i, k)$ must be consecutive, with any 0 's lying to the left or right end points of this string.

\subsection{Containment}

The condition of containment states that regions in $A$ must contain 12 distinct pitch classes. Let $B_{p}(0 \leq p \leq 11)$ be the set of all locations $(i, j)$ of pitch class $p$ in matrix $A$. From this, we can express the condition of containment by the following:

$$
\forall p \in[0,11], \forall k \in[1, K], \sum_{(i, j) \in B_{p}} x_{i, j, k}=1,
$$

where for each $k, x_{i, j, k}$ is equal to 1 at one and only one location $(i, j)$ whose pitch class is $p$ in $A$. When this is the case, $C_{k}$ will contain one of each pitch class.

\subsection{Covering all $(i, j)$ in $A$}

A solution to our problem requires that every one element in $A$ is covered by at least one of the regions, $C_{k}$. We can express this condition by the following constraint:

$$
\forall i \in[1, I], \forall j \in[1, J], \underset{k=1}{K}\left(x_{i, j, k}=1\right)
$$

\subsection{Restrictions on the left-to-right order of candidate sets and their overlaps}

As discussed in section 1 , adjacent regions need not be contiguous in each row in $A$, however, there are restrictions on their left-to-right order and allowed 
overlaps. The number of overlaps in each row between these regions must not exceed 1 . We can express this restriction by the following constraint:

$$
\begin{aligned}
\forall i \in[1, I], \forall j \in[2, J], \forall k \in[1, K-1], \\
\left(x_{i, j, k}=1\right) \Longrightarrow \bigwedge_{k^{\prime}=k+1}^{K}\left(x_{i, j-1, k^{\prime}}=0\right) .
\end{aligned}
$$

When combined with the constraint of consecutiveness, constraint 5 means that if $x_{i, j, k}$ is equal to 1 , the $i$ th row of $C_{k^{\prime}}$, whose $k^{\prime}$ is greater than $k$, is either (1) located at the right-hand side of $(i, j)$ without overlapping the $i$ th row of $C_{k}$ or (2) has only one overlap at the right-most element of the $i$ th row of $C_{k}$.

\subsection{Candidate sets as all different integer partitions}

In order to determine that the integer composition "shape" of $C_{k}$ is a distinct integer partition, we introduce two variables, $y_{i, k, l}$ and $z_{k, l}$. Let $y_{i, k, l}$ be a binary variable that indicates whether or not the length of the $i$ th row of $C_{k}$ is greater than or equal to $l(1 \leq l \leq L, L=12)$, by introducing the following two constraints:

$$
\begin{gathered}
\forall i \in[1, I], \forall k \in[1, K], \sum_{j=1}^{J} x_{i, j, k}=\sum_{l=1}^{L} y_{i, k, l} \\
\forall i \in[1, I], \forall k \in[1, K], \forall l \in[2, L],\left(y_{i, k, l}=1\right) \Longrightarrow\left(y_{i, k, l-1}=1\right) .
\end{gathered}
$$

Equation 6 states that the sum of all elements in $\left\langle y_{i, k, 1}, y_{i, k, 2}, \ldots, y_{i, k, L}\right\rangle$ is equal to the length of the $i$ th row of $C_{k}$ while Equation 7 states that its elements equal to 1 begin in the first position and are consecutive. For example, when the length of the $i$ th row of $C_{k}$ is $3,\left\langle y_{i, k, 1}, y_{i, k, 2}, \ldots, y_{i, k, L}\right\rangle$ is $\langle 1,1,1,0,0,0,0,0,0,0,0,0\rangle$. The total number of rows in $C_{k}$ whose lengths are greater than or equal to $l$ is given by $\sum_{i=1}^{I} y_{i, k, l}$. Let $z_{k, l}\left(0 \leq z_{k, l} \leq I\right)$ be an integer variable that is equal to $\sum_{i=1}^{I} y_{i, k, l}(1 \leq l \leq L)$ with the following constraint:

$$
\forall k \in[1, K], \forall l \in[1, L], z_{k, l}=\sum_{i=1}^{I} y_{i, k, l} .
$$

The ordered set of twelve values $z_{k, l}(1 \leq l \leq L)$ will then identify the type of integer partition corresponding to $C_{k}$. For example, when $z_{k, l}$ is $\langle 4,4,2,1,1,0,0,0,0,0,0,0,0\rangle, C_{k}$ is $\operatorname{IntPart}_{12}(5,3,2,2)$. We denote this set $z_{k, l}$ corresponding to an integer partition $n$ by $P_{n}=\left\langle P_{n, 1}, P_{n, 2}, \ldots P_{n, L}\right\rangle(1 \leq n \leq$ $N, N=34$ ), where integer partitions appear in reverse lexicographical order, meaning that those containing the fewest parts and largest part lengths appear first. For example, $P_{1}$ is $\operatorname{IntPart}_{12}(12,0,0,0)$ and $P_{34}$ is $\operatorname{IntPart}_{12}(3,3,3,3)$. From this, we determine the integer composition shape of $C_{k}$ to be the integer partition $n$ by the following constraint:

$$
\forall k \in[1, K], \forall n \in[1, N],\left(w_{k}=n\right) \Longleftrightarrow \wedge_{l=1}^{L}\left(z_{k, l}=P_{n, l}\right),
$$


where $w_{k}\left(1 \leq w_{k} \leq N\right)$ is an integer variable that indicates to which $P_{n} C_{k}$ corresponds. We can now express the condition that all integer partitions are distinct by the constraint, $\operatorname{All} \operatorname{Different}\left(w_{1}, w_{2}, \ldots, w_{K}\right)$.

\section{Solution}

In order to confirm that our formulation of this problem is accurate, we implemented our constraints described in section 2 and supplied these to a CP solver (Sugar v2-1-0 [12,13]). We first tried to solve for the whole matrix directly, however, we were unable to obtain a solution after a day of calculation. We decided instead, to divide the matrix into two, equally-sized halves and try solving for each in such a way that their re-joining would form a complete solution to the original problem. We made this division of the original matrix at $[1, I] \times[1, J / 2]$. Columns 1 to $(J / 2)$ then correspond to the first smaller matrix we denote by $A_{1}$ and columns $(J / 2)+1$ to $J$ correspond to the second smaller matrix we denote by $A_{2}$. We allocated $34 / 2=17$ integer partitions to be found in each.

With little modification, our constraints can be adapted to the solving of these sub-problems. These changes include modifying $B_{p}$ (in equation 3 ) to contain only the locations of pitch classes in either $A_{1}$ or $A_{2}$, setting $K$ to be the new number of partitions in each (i.e., 17 ) and $J$ to be their new column lengths $96 / 2=48$. Solutions to $A_{1}$ and $A_{2}$ in which no integer partition is used more than once and contains only pitch classes from one or the other matrix (but not both), collectively form a solution to the original problem. Due to its smaller size, we were able to find solutions beginning with $A_{1}$ over the course of a day, in which 506 were found. Naturally, solving for $A_{1}$ makes finding a solution in $A_{2}$ more difficult as the number of available partitions is now fewer, and in fact, all 506 solutions to $A_{1}$ made $A_{2}$ unsatisfiable. We noticed, however, that certain partitions in these 506 solutions e.g., $\operatorname{IntPart}_{12}(3,3,3,3)$ and $\operatorname{IntPart}_{12}(4,3,3,2)$ occurred far less frequently than others. It would be reasonable then to conclude that solutions in $A_{1}$ which contain the greatest number of these less frequently occurring partitions will make solving for $A_{2}$ more likely, as the fewer available partitions in $A_{2}$ now consist of a proportionally greater number of frequently occurring partitions. Therefore, we solved again for $A_{1}$, this time by arbitrarily restricting the domain of $w_{k}$ to exclude the top 6 most frequently occurring partitions and include the top 5 least frequently occurring partitions.

If we denote the subset of integers from $[1,34]$ corresponding to the partitions found in this solution to $A_{1}, S$, then the domain of $w_{k}$ for possible solutions to $A_{2}$ becomes $[1,34] \backslash S$. We then tried solving for $A_{2}$, under the assumption that its proportionally greater number of more frequently occurring partitions would make finding a solution easier. While this means we exclude possible solutions e.g., ones in which a rarely occurring partition occurs in $A_{2}$ or where a partition contains pitch classes from both $A_{1}$ and $A_{2}$, we were able to generate a complete solution in this way. Solving for $A_{1}$ took approx. 4 minutes while solving for $A_{2}$ took approx. 28 minutes. Table 1 shows the complete solution found using this method of re-joining $A_{1}$ and $A_{2}$. 


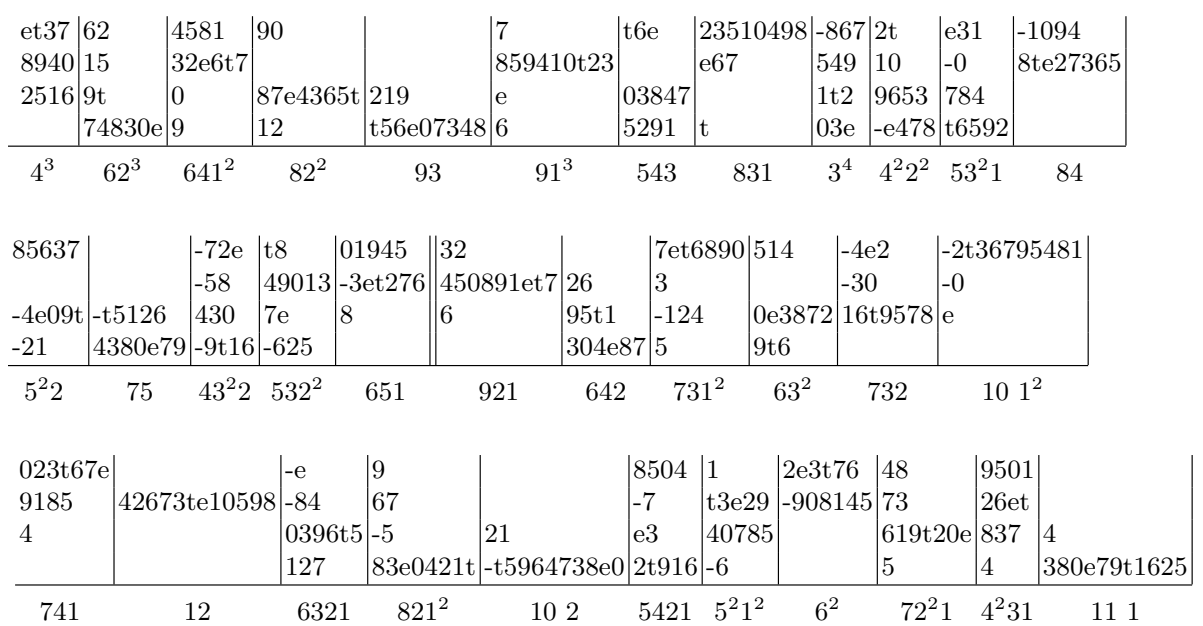

Table 1: A generated all-partition array corresponding to a complete solution to our problem, represented in the way used by music theorists [2]. Each column contains the elements in $A$ belonging to $C_{k}$, where a dash indicates those that overlap. Note, that partitions are denoted using a shorthand notation, e.g., $4^{3}$, where the base indicates the length of a part and the exponent denotes its number of occurrences. For clarity, the integers 10 and 11 have been replaced by the letters $t$ and e, respectively.

\section{Conclusion}

In this paper, we have introduced a novel formulation of one part of the problem of generating an all-partition array, beginning from a given matrix, using constraint programming $(\mathrm{CP})$. Solving for the whole of this matrix directly proved too difficult using our constraints. Therefore, we introduced a method of dividing the matrix into two halves, solving for each and then re-joining them to form a complete solution. Using this method, we were able to discover a solution. This solution is the first we are aware of to be automatically generated by a computer. Moreover, it is an all-together new all-partition array from those previously discovered by Babbitt and other composers. In future work, we hope to examine in more detail how to make finding solutions in larger matrices possible and without excluding potential solutions.

Acknowledgments. The work of Tsubasa Tanaka reported in this paper was supported by JSPS Postdoctoral Fellowships for Research Abroad. The work of Brian Bemman and David Meredith was carried out as part of the project Lrn2Cre8, which acknowledges the financial support of the Future and Emerging Technologies (FET) programme within the Seventh Framework Programme for Research of the European Commission, under FET grant number 610859. 


\section{References}

1. Babbitt, M.: Since Schoenberg. Perspectives of New Music, 12(1/2), 3-28 (1973).

2. Mead, A.: An Introduction to the Music of Milton Babbitt. Princeton University Press, Princeton, NJ, (1994).

3. Anders T., Anagnostopoulou, C., and Alcorn M.: Strasheela: Design and Usage of a Music Composition Environment Based on the Oz Programming Model. In Roy, P. editor, Multiparadigm Programming in Mozart/OZ: Second International Conference, MOZ 2004, LNCS 3389, Springer-Verlag, (2005).

4. Laurson, M. and Kuuskankare, M.: A constraint based approach to musical textures and instrumental writing. In Proceedings of the 7 th International Conference on Principles and Practice of Constraint Programming, Musical Constraints Workshop, (2001).

5. Carpentier, G., Assayag, G. and Saint-James, E.: Solving the Musical Orchestration Problem using Multiobjective Constrained Optimization with a Genetic Local Search Approach. Heuristics 16(5), 681-714, Springer, (2010).

6. Chemillier, M. and Truchet, C.: Two musical CSPs. In Proceedings of the 7th International Conference on Principles and Practice of Constraint Programming, Musical Constraints Workshop, (2001).

7. Puget, J. F. and Régin J. C.: Solving the All Interval Problem https://ianm.host. cs.st-andrews.ac.uk/CSPLib/prob/prob007/puget.pdf

8. Bemman, B. and Meredith, D.: Generating Milton Babbitt's all-partition arrays. Journal of New Music Research, 45(2), (2016a). http://www.tandfonline.com/ $\mathrm{doi} / \mathrm{full} / 10.1080 / 09298215.2016 .1172646$

9. Starr, D. and Morris, R.: A general theory of combinatoriality and the aggregate, part 1. Perspectives of New Music, 16(1), 3-35 (1977).

10. Starr, D. and Morris, R.: A general theory of combinatoriality and the aggregate, part 2. Perspectives of New Music, 16(2), 50-84 (1978).

11. Bazelow, A. R. and Brickle, F.: A combinatorial problem in music theory: Babbitt's partition problem (I). Annals of the New York Academy of Sciences, 319(1), 47-63 (1979).

12. http://bach.istc.kobe-u.ac.jp/sugar/

13. Naoyuki, T. and Mutsunori B.: Sugar: A CSP to SAT Translator Based on Order Encoding, in Proceedings of the 2nd International CSP Solver Competition, 65-69 (2008). 